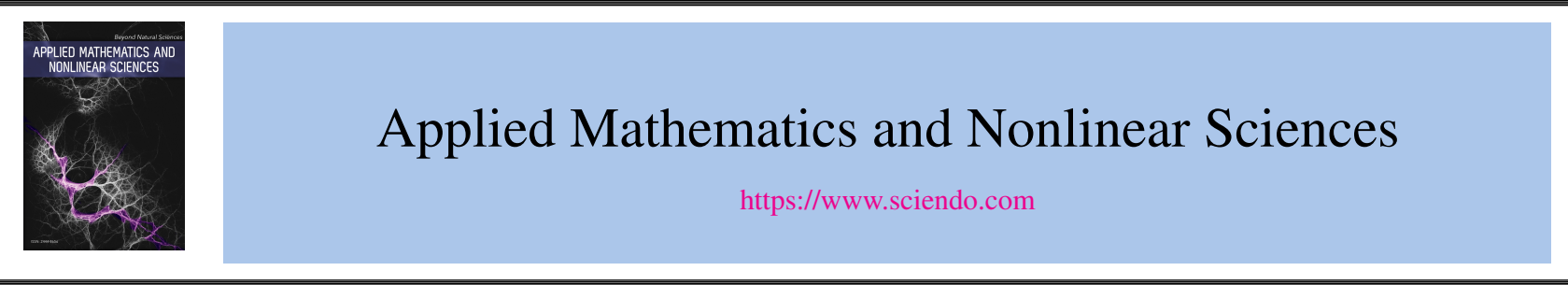

\title{
Deterministic chaos in pendulum systems with delay
}

\author{
Aleksandr Shvets ${ }^{\dagger}$, Alexander Makaseyev \\ National Technical University of Ukraine "Igor Sikorsky Kyiv Polytechnic Institute",
} Ukraine

Submission Info

Communicated by Juan Luis García Guirao

Received November 13th 2018

Accepted January 12th 2019

Available online February 12th 2019

\begin{abstract}
Dynamic system "pendulum - source of limited excitation" with taking into account the various factors of delay is considered. Mathematical model of the system is a system of ordinary differential equations with delay. Three approaches are suggested that allow to reduce the mathematical model of the system to systems of differential equations, into which various factors of delay enter as some parameters. Genesis of deterministic chaos is studied in detail. Maps of dynamic regimes, phase-portraits of attractors of systems, phase-parametric characteristics and Lyapunov characteristic exponents are constructed and analyzed. The scenarios of transition from steady-state regular regimes to chaotic ones are identified. It is shown, that in some cases the delay is the main reason of origination of chaos in the system "pendulum - source of limited excitation".
\end{abstract}

Keywords: chaotic attractor, system with limited excitation, factors of delay.

AMS 2010 codes: 34C15, 37D45.

\section{Introduction}

In mathematical modeling of oscillatory processes a mathematical model of a relatively simple dynamical system is often used to study the dynamics of much more complex systems. Typical example of this approach is the extensive use of pendulum models to study the dynamics of systems of an entirely different nature. Pendulum mathematical models are widely used to describe the dynamics of various technical constructions, machines and mechanisms, in the study of cardiovascular system, financial markets, etc. Such widespread use of pendulum models makes it relevant to study directly the dynamics of pendulum systems.

Modern development of energy efficient and energy-preserving technologies requires the highest minimization of excitation source power of oscillatory systems. This leads to the fact that the energy of excitation source

†Corresponding author.

Email address: alex.shvets@bigmir.net

\section{sciendo}


is comparable to the energy consumed by the oscillating system. Such systems as "source of excitation - oscillating subsystem" are called non-ideal by Kononenko [1]. In mathematical modeling of such systems, the limitation of excitation source power must be always taken into account.

Another important factor that significantly affects the change of steady-state regimes of dynamical systems, is the presence of different in their physical substance, factors of delay. The factors of delay are always present in rather extended systems due to the limitations of signal transmission speed: stretching, waves of compression, bending, current strength, etc. In some cases, taking into account factors of delay leads only to minor quantitative changes in dynamical characteristics of pendulum systems. In other cases, taking into account these factors allow to identify qualitative changes in dynamical characteristics [2,3].

The study of the influence of the factors of delay on the dynamical stability of equilibrium positions of pendulum systems was initiated by Shvets and Mitropolsky - and his scientific school - in the 1980s [4]. But only ideal pendulum models were initially considered. In this paper non-ideal pendulum systems of the type "pendulum - electric motor" are considered. Mathematical models of such non-ideal systems were obtained in [5]. In such systems the existence of chaotic attractors was discovered and proved that the main cause of chaos is limited excitation [5]. The aim of this work is to study the influence of various factors of delay on dynamical behaviour of these system.

\section{Mathematical model of the system}

In [5] the equations of motion of the system "pendulum - electric motor" in the absence of any factors of delay were obtained. They are

$$
\left\{\begin{array}{l}
\frac{d y_{1}}{d \tau}=C y_{1}-y_{2} y_{3}-\frac{1}{8}\left(y_{1}^{2} y_{2}+y_{2}^{3}\right) \\
\frac{d y_{2}}{d \tau}=C y_{2}+y_{1} y_{3}+\frac{1}{8}\left(y_{1}^{3}+y_{1} y_{2}^{2}\right)+1 \\
\frac{d y_{3}}{d \tau}=D y_{2}+E y_{3}+F
\end{array}\right.
$$

where phase variables $y_{1}, y_{2}$ describe the deviation of the pendulum from the vertical and phase variable $y_{3}$ is proportional to the rotation speed of the motor shaft. The system parameters are defined by

$$
C=-\delta_{1} \varepsilon^{-2 / 3} \omega_{0}^{-1}, D=-\frac{2 m l^{2}}{I}, F=2 \varepsilon^{-2 / 3}\left(\frac{N_{0}}{\omega_{0}}+E\right),
$$

where $m$ - the pendulum mass, $l$ - the reduced pendulum length, $\omega_{0}$ - natural frequency of the pendulum, $a$ - the length of the electric motor crank, $\varepsilon=\frac{a}{l}, \delta_{1}$ - damping coefficient of the medium resistance force, $I$ - the electric motor moment of inertia, $E, N_{0}$ - constants of the electric motor static characteristics.

Let us consider the following system of equations:

$$
\left\{\begin{array}{l}
\frac{d y_{1}(\tau)}{d \tau}=C y_{1}(\tau-\delta)-y_{2}(\tau) y_{3}(\tau-\gamma)-\frac{1}{8}\left(y_{1}^{2}(\tau) y_{2}(\tau)+y_{2}^{3}(\tau)\right) \\
\frac{d y_{2}(\tau)}{d \tau}=C y_{2}(\tau-\delta)+y_{1}(\tau) y_{3}(\tau-\gamma)+\frac{1}{8}\left(y_{1}^{3}(\tau)+y_{1}(\tau) y_{2}^{2}(\tau)\right)+1 \\
\frac{d y_{3}(\tau)}{d \tau}=D y_{2}(\tau-\gamma)+E y_{3}(\tau)+F
\end{array}\right.
$$

This system is a system of equations with constant delay. Positive constant parameter $\gamma$ was introduced to account the delay effects of electric motor impulse on the pendulum. We assume that the delay of the electric 
motor response to the impact of the pendulum inertia force is also equal to $\gamma$. Taking into account the delay $\gamma$ conditioned by the fact that the wave velocity perturbations on the elements of the construction has a finite value that depends on the properties of external fields, for instance, the temperature field. In turn, the constant positive parameter $\delta$ characterizes the delay of the medium reaction on the dynamical state of the pendulum. This delay is due to the limited sound velocity in that medium.

Let us consider two approaches that allow to reduce the time-delay system (3) to the systems of equations without delay. The first approach is as follows. Assuming a small delay, we can write

$$
\begin{aligned}
& y_{i}(\tau-\gamma)=y_{i}(\tau)-\frac{d y_{1}(\tau)}{d \tau} \gamma+\ldots, \quad i=2,3 . \\
& y_{i}(\tau-\delta)=y_{i}(\tau)-\frac{d y_{1}(\tau)}{d \tau} \delta+\ldots, \quad i=1,2 .
\end{aligned}
$$

Then, if $C \delta \neq-1$, we get the following system of equations:

$$
\left\{\begin{array}{l}
\dot{y_{1}}=\frac{1}{1+C \delta}\left(C y_{1}-y_{2}\left[y_{3}-\gamma\left(D y_{2}+E y_{3}+F\right)\right]-\frac{1}{8}\left(y_{1}^{2} y_{2}+y_{2}^{3}\right)\right) \\
\dot{y_{2}}=\frac{1}{1+C \delta}\left(C y_{2}+y_{1} y_{3}-y_{1} \gamma\left(D y_{2}+E y_{3}+F\right)+\frac{1}{8}\left(y_{1}^{3}+y_{1} y_{2}^{2}\right)+1\right) \\
\dot{y_{3}}=(1-C \gamma) D y_{2}-\frac{D \gamma}{8}\left(y_{1}^{3}+y_{1} y_{2}^{2}+8 y_{1} y_{3}+8\right)+E y_{3}+F
\end{array}\right.
$$

The obtained system of equations is already a system of ordinary differential equations. Delays are included in this system as additional parameters.

In order to approximate the system (3) another, more precise, method can be used [6]. If $\gamma>0, \delta>0$ let us divide the segments $[-\gamma ; 0]$ and $[-\delta ; 0]$ into $m$ equal parts. We introduce the following notation

$$
\begin{aligned}
& y_{1}\left(\tau-\frac{i \delta}{m}\right)=y_{1 i}(\tau), y_{2}\left(\tau-\frac{i \gamma}{m}\right)=y_{2 i}(\tau), y_{2}\left(\tau-\frac{i \delta}{m}\right)=\tilde{y}_{2 i}(\tau), \\
& y_{3}\left(\tau-\frac{i \gamma}{m}\right)=y_{3 i}(\tau), i=\overline{0, m} .
\end{aligned}
$$

Then, using difference approximation of derivative [6], [7] we obtain

$$
\left\{\begin{array}{l}
\frac{d y_{10}(\tau)}{d \tau}=C y_{1 m}(\tau)-y_{20}(\tau) y_{3 m}(\tau)-\frac{1}{8}\left(y_{10}^{2}(\tau) y_{20}(\tau)+y_{20}^{3}(\tau)\right) \\
\frac{d y_{20}(\tau)}{d \tau}=C \tilde{y}_{2 m}(\tau)+y_{10}(\tau) y_{3 m}(\tau)+\frac{1}{8}\left(y_{10}^{3}(\tau)+y_{10}(\tau) y_{20}^{2}(\tau)\right)+1 \\
\frac{d y_{30}(\tau)}{d \tau}=D y_{2 m}(\tau)+E y_{30}(\tau)+F \\
\frac{d y_{1 i}(\tau)}{d \tau}=\frac{m}{\delta}\left(y_{1 i-1}(\tau)-y_{1 i}(\tau)\right), \quad i=\overline{1, m} \\
\frac{d y_{2 i}(\tau)}{d \tau}=\frac{m}{\gamma}\left(y_{2} i-1(\tau)-y_{2 i}(\tau)\right), \quad i=\overline{1, m} \\
\frac{d \tilde{y}_{2 i}(\tau)}{d \tau}=\frac{m}{\delta}\left(\tilde{y}_{2} i-1(\tau)-\tilde{y}_{2 i}(\tau)\right), \quad i=\overline{1, m} \\
\frac{d y_{3 i}(\tau)}{d \tau}=\frac{m}{\gamma}\left(y_{3 i-1}(\tau)-y_{3 i}(\tau)\right), \quad i=\overline{1, m}
\end{array}\right.
$$

It is a system of ordinary differential equations of $(4 m+3)$-th order. In the absence of one of the delays $(\gamma$ or $\delta$ ), using the same reasoning, the system (3) can be reduced to the systems of $(2 m+3)$-th order. As in the system (4), the delays are included in these systems as additional parameters. 
Choosing a sufficiently large $m$ in the system (5), the system (3) will be very well approximated by the system (5) [6]. We note that the solutions $y_{1}, y_{2}, y_{3}$ of the system (3) are described by the functions $y_{10}, y_{20}, y_{30}$ of the system (5).

Therefore, we obtained three-dimensional (4) and fifteen-dimensional (5) models each describing the system of equations with delay (3). These models are the systems of non-linear differential equations, so in general the study of steady-state regimes can be carried out only by using numerical methods and algorithms. The methodology of such studies is described in detail in [5].

\section{Maps of dynamical regimes}

For general analysis of nonlinear dynamical behaviour the maps of dynamical regimes are constructed. Such maps provide a crucial information about the type of steady-state regime of the system depending on its parameters. The construction of dynamical regimes maps is based on analysis and processing of spectrum of Lyapunov characteristic exponents [5, 8]. Where necessary, for more accurate determination of steady-state regime of the system, we study other characteristics of attractors: phase portraits, Poincare sections and maps, Fourier spectrums and distributions of the invariant measure.

Let us consider the behavior of the systems (4) and (5) when the parameters are $C=-0.1, D=-0.53$, $E=-0.59, F=-0.4$. The map of dynamical regimes in fig. 1 (a) was built for three-dimensional model (4) and the map in fig. 1 (b) was built for fifteen-dimensional model (5). These figures illustrate the effect of the delay of interaction between pendulum and electric motor $\gamma$ and the delay of the medium $\delta$ on changing the type
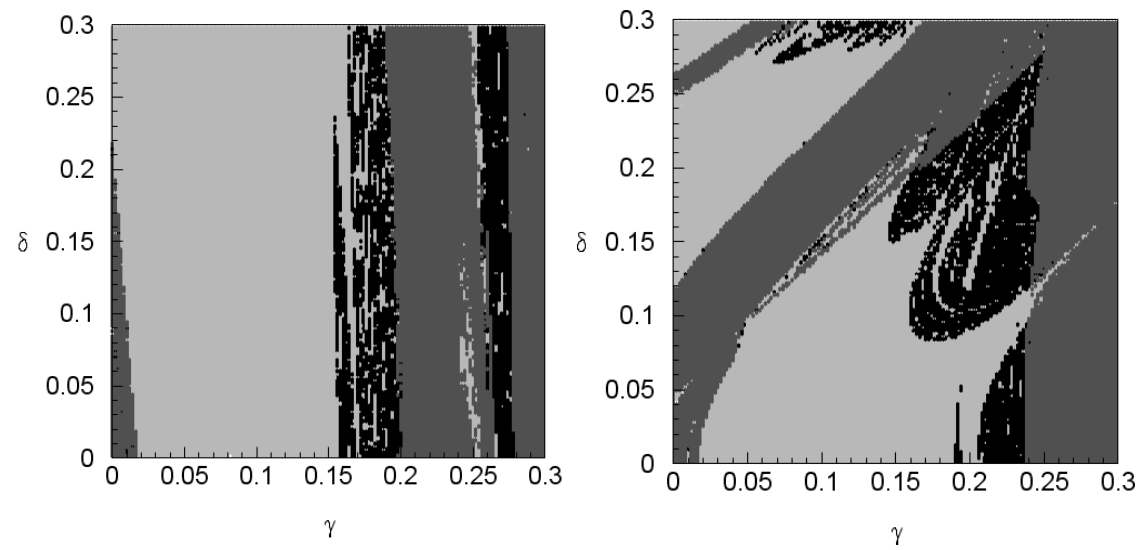

a
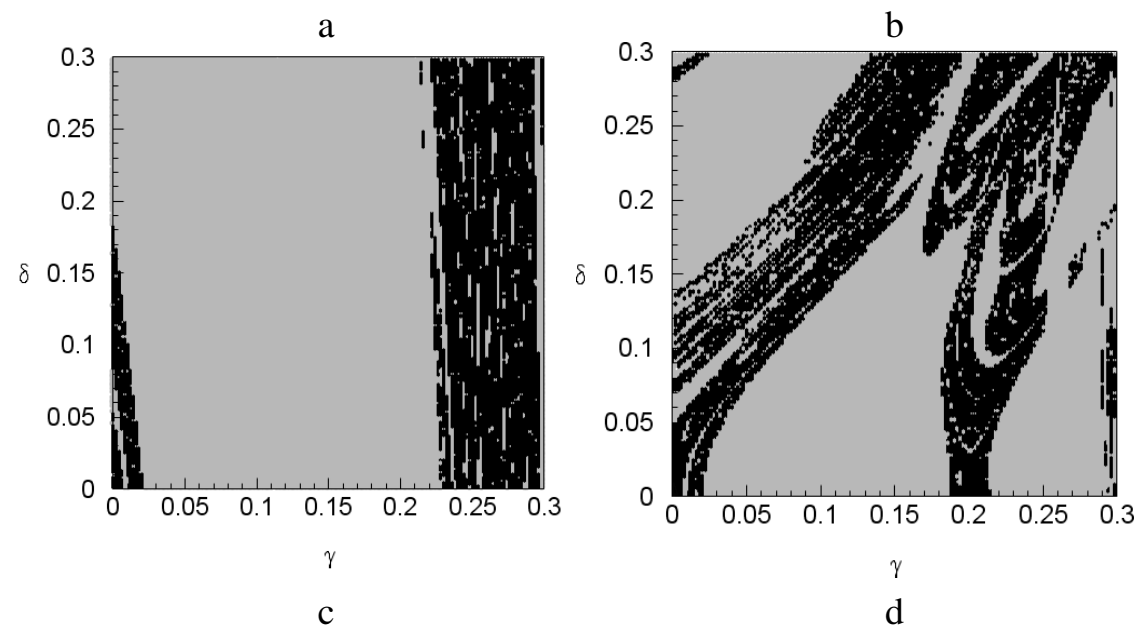

Fig. 1 Maps of dynamical regimes 
of steady-state regime of the systems. The dark-grey areas of the maps correspond to equilibrium positions of the system. The light-grey areas of the maps correspond to limit cycles of the system. And finally, the black areas of the maps correspond to chaotic attractors.

At small values of the delays both systems have stable equilibrium positions (dark-grey areas in the figures). With an increase of the delay values the region of stable equilibrium positions is replaced by the region of periodic regimes and then by the region of chaotic regimes. With further increase of the delays the alternation of these three types of dynamical regimes takes place.

Let us study the dynamics of the system (4) and (5) at other values of the parameters. The maps of dynamical regimes of three-dimensional system (4) and fifteen-dimensional system (5) at $C=-0.1, D=-0.5, E=-0.59$, $F=-0.31$ are built respectively in fig. 1 (c), (d). As seen from the constructed maps, at small values of the delays $\gamma$ and $\delta$ both systems systems have chaotic attractors (black areas in the maps). With an increase of the delay values the region of chaotic regimes is replaced by a large area of periodic steady-state regimes. In the map of dynamical regimes (fig. 1 (d)) of the fifteen-dimensional system (5) in the area of limit cycles there is a sufficiently large region of chaotic regimes. In three-dimensional system (fig. 1 (c)) such region does not exist.
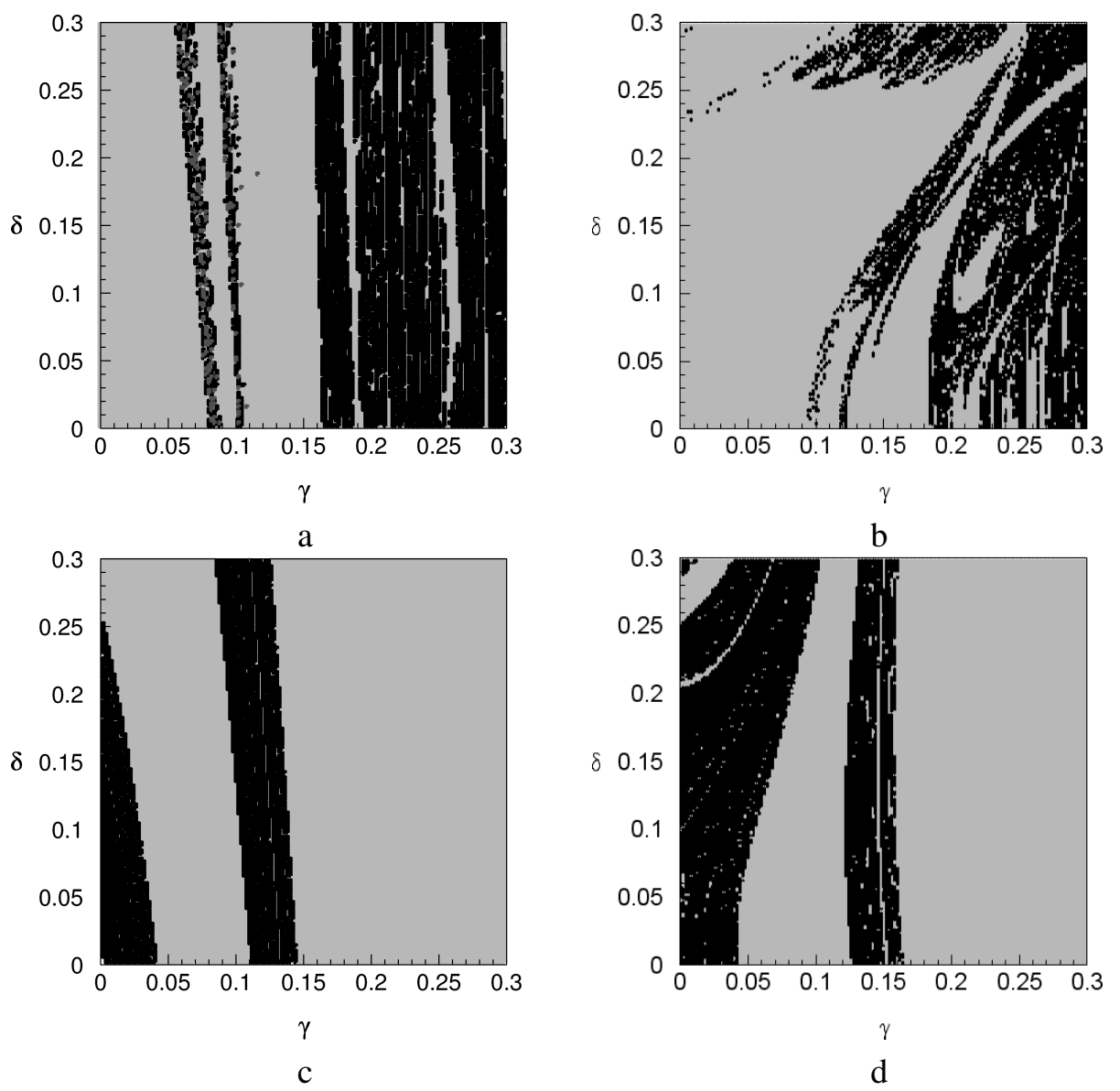

Fig. 2 Maps of dynamical regimes

The maps of dynamical regimes of respectively the system (4) and the system (5) at $C=-0.1, D=-0.6$, $E=-0.7, F=-0.4$ are built in fig. 2 (a), (b). At small values of the delays the steady-state regime of both systems is limit cycle (light-grey areas in the figures). With an increase of the delay values the maps in fig. 2 (a), (b) are certainly different. In fig. 2 (a) there are narrow area in which the limit cycle is replaced by an equilibrium position, as well as by a chaotic attractor. Whereas in fig. 2 (b) these narrow area is almost missing. Further in both figures there are a rather wide area of periodic regimes, which with further increase of the delay 
is replaced by chaos area. Moreover, in this rather wide area of chaos fairly narrow strips of periodic regimes are built in.

In fig. 2 (c), (d) the maps of dynamical regimes of respectively the system (4) and the system (5) at $C=-0.1$, $D=-0.53, E=-0.6, F=0.19$ are constructed. At small values of the delays both systems have chaotic attractors (black areas in the figures). With an increase of the delay values the region of chaos is replaced by the region of periodic regimes. Then again chaos arises in the system. Further this area is replaced by the area of limit cycles.

As seen from the constructed maps of dynamical regimes, the dynamics of three-dimensional system (4) and fifteen-dimensional system (5) is the same only at small values of the delay $\gamma$ and $\delta$. With an increase of the delays the dynamical behaviour of these systems is significantly different.

The obtained maps of dynamical regimes allow us to conduct a quick qualitative identification of the type of steady-state regime of the systems (4) and (5). On the basis of constructed maps, more detailed studies of emerging dynamical regimes can be carried out. Particularly we can study the transition from regular to chaotic regimes.

\section{Regular and chaotic dynamics}

Let us study the types of regular and chaotic attractors that exist in the systems (4) and (5). We implement a horizontal section of the maps of dynamical regimes in fig. 2 (c), (d) along the delay $\gamma$ at $\delta=0.15$. In other words, let us consider the behavior of the systems (4) and (5) when parameters are $C=-0.1, D=-0.53$, $E=-0.6, F=0.19$ and the delays $\delta=0.15$ and $0 \leq \gamma \leq 0.3$.

In fig. 3 (a), (b) the dependence of maximum non-zero Lyapunov characteristic exponent on $\gamma$ and phaseparametric characteristic of three-dimensional system (4) are shown respectively. These figures illustrate the influence of the delay of interaction between pendulum and electric motor $\gamma$ on chaotization of the system (4).

Let us construct the same characteristics at the same values of the parameters for fifteen-dimensional system (5). In fig. 3 (c), (d) respectively the dependence of maximum non-zero Lyapunov characteristic exponent on $\gamma$ and phase-parametric characteristic are shown.

In fig. 3 (a), (c) we can clearly see the presence of intervals $\gamma$ in which maximum Lyapunov exponent of the systems is positive. In these intervals the systems have chaotic attractors. The area of existence of chaos is clearly seen in phase-parametric characteristics of the systems. The areas of chaos in the bifurcation trees are densely filled with points. A careful examination of the obtained images allow not only to identify the origin of chaos in the systems, but also to describe the scenario of transition to chaos. So with a decrease of $\gamma$ there are the transitions to chaos by Feigenbaum scenario (infinite cascade of period-doubling bifurcations of a limit cycle). Bifurcation points for the delay $\gamma$ are clearly visible in each figures. These points are the points of approaches of the Lyapunov exponent graph to the zero line (fig. 3 (a), (c)) and the points of splitting the branches of the bifurcation tree (fig. 3 (b), (d)). In turn, the transition to chaos with an increase of the delay happens under the scenario of Pomeau-Manneville, in a single bifurcation, through intermittency.

A careful analysis of these figures allows to see qualitative similarity of the respective characteristics of the systems (4) and (5). However, with increasing the delay the differences in the dynamics of these systems become very significant.

For instance when the delay of interaction between pendulum and electric motor $\gamma=0.05$ and the delay of the medium $\delta=0.15$ the steady-state regime of three-dimensional system (4) is periodic and the attractor is limit cycle. Phase portrait of this attractor is shown in fig. 4 (a). Whereas at this values of the parameters and the delays fifteen-dimensional system (5) has steady-state chaotic dynamical regime. Phase portrait of the chaotic attractor of the system (5) is built in fig. 4 (b).

It is also possible a different situation. For instance at the delays $\gamma=0.11, \delta=0.15$ the system (4) has chaotic steady-state regime of oscillations. Phase portrait of the attractor is shown in fig. 4 (c). Whereas at this values of the delay fifteen-dimensional system (4) has regular periodic dynamical regime and its attractor is limit 
cycle (fig. 4 (d)).

This suggests that three-dimensional system of equations (4) should be used to study the system (3) only at very small values of the delay. With increasing values of the delay to study regular and chaotic oscillations of "pendulum - electric motor" system, fifteen-dimensional system of equations (5) should be used.

\section{Conclusion}

Taking into account various factors of delay in non-ideal pendulum systems is crucial. The presence of delay in such systems can affect the qualitative change in the dynamical behaviour. It is shown, that in some cases the delay is the main reason of origination as well as vanishing of chaotic attractors.

It is shown that for small values of the delay it is sufficient to use three-dimensional mathematical model, whereas for relatively high values of the delay the fifteen-dimensional mathematical model should be used.
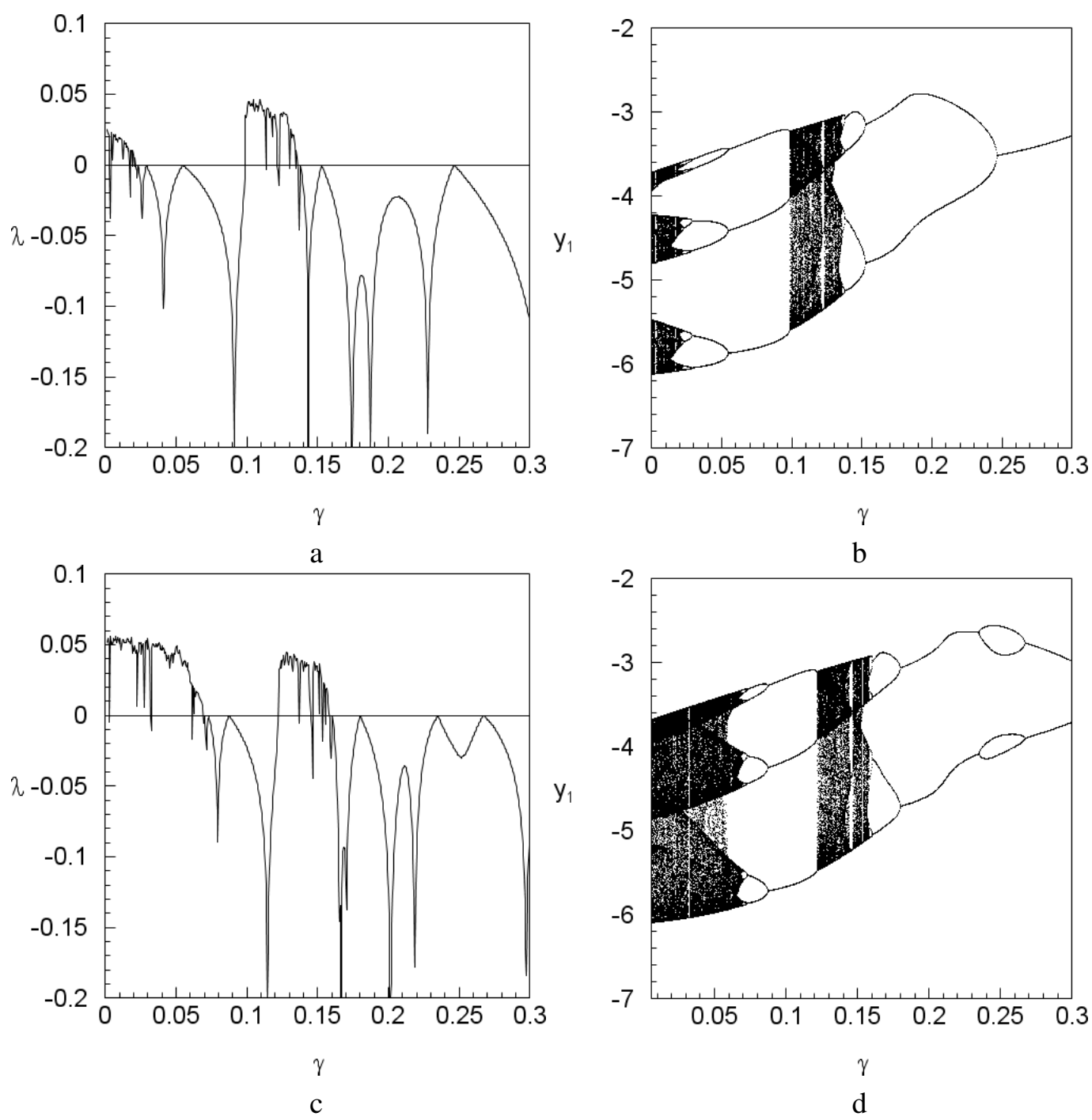

Fig. 3 The dependencies of maximal non-zero Lyapunov characteristic exponent on $\gamma(\mathrm{a})$, (c), phase-parametric characteristics (b), (d) 

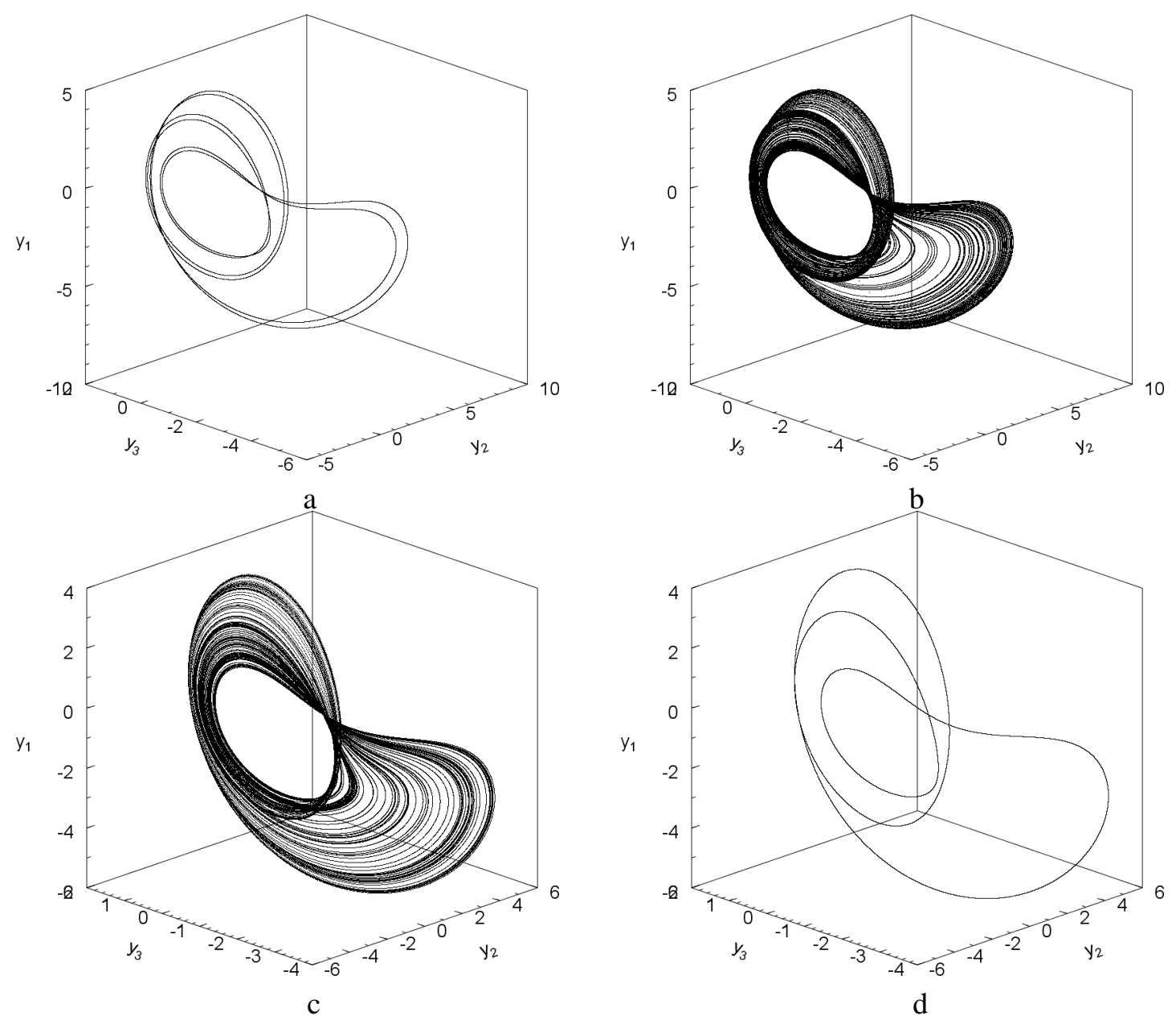

Fig. 4 Phase portraits of attractors of the system (4) (a), (c) and the system (5) (b), (d)

\section{References}

[1] V. O. Kononenko, 1. (1969), Vibrating System with a Limited Power-supply, Iliffe, London.

[2] A. Yu. Shvets and A. M. Makaseyev, 2. (2014), Mathematical Simulation of the Influence of Delay Factors on the Oscillations of Non-ideal Pendulum Systems, Proceedings of Institute of Mathematics of National Academy of Sciences of Ukraine. Vol. 11, Number 5. P. 269-297.

[3] A. Yu. Shvets and A. M. Makaseyev, 3. (2014), Chaos in Pendulum Systems with Limited Excitation in the Presence of Delay. Chaotic Modeling and Simulation (CMSIM), 3:233-241, 2014.

[4] A. Yu. Shvets and Yu. A. Mitropolsky, 4. (1980), About influence of delay on a stability of a pendulum with the vibrating suspension point. Analytical methods of non-linear oscillations. Kyiv, P. 115-120.

[5] T. S. Krasnopolskaya and A. Yu. Shvets, 5. (2008), Regular and chaotical dynamics of systems with limited excitation. R\&C Dynamics, Moscow.

[6] N. A. Magnizkiy and S. V. Sidorov, 6. (2004), New methods of chaotic dynamics. Editorial URSS.

[7] A. A. Samarskiy and A. V. Gulin, 7. (1989), Numerical methods. Nauka.

[8] S. P. Kouznetsov, 8. (2001), Dynamic chaos. Physmatlit, Moscow. 